\title{
Correction to: Recommendations for the management of rheumatoid arthritis in the Eastern Mediterranean region: an adolopment of the 2015 American College of Rheumatology guidelines
}

Thurayya Arayssi ${ }^{1}$ - Manale Harfouche ${ }^{2} \cdot$ Andrea Darzi $^{3} \cdot$ Samar Al Emadi ${ }^{4} \cdot$ Khalid A. Alnaqbi $^{5} \cdot$ Humeira Badsha $^{6}$. Farida Al Balushi ${ }^{7}$. Carole Dib ${ }^{8}$. Bassel Elzorkany ${ }^{9} \cdot$ Hussein Halabi $^{10}$. Mohammed Hammoudeh ${ }^{4}$. Wissam Hazer ${ }^{11}$. Basel Masri ${ }^{12}$. Mira Merashli ${ }^{13} \cdot$ Mohammed Omair $^{14} \cdot$ Nelly Salloum $^{8} \cdot$ Imad Uthman $^{13} \cdot$ Sumeja Zahirovic $^{1}$. Nelly Ziade ${ }^{15}$. Raveendhara R. Bannuru ${ }^{16}$. Timothy McAlindon ${ }^{16}$. Mohamed A. Nomier ${ }^{17}$. Jasvinder A. Singh ${ }^{18,19}$. Robin Christensen $^{20,21}$ - Peter Tugwell ${ }^{22}$ - Holger Schünemann ${ }^{23}$ • Elie A. Akl ${ }^{13,23,24}$

Published online: 6 September 2018

(C) International League of Associations for Rheumatology (ILAR) 2018

Correction to: Clinical Rheumatology

https://doi.org/10.1007/s10067-018-4245-5

The original version of this article, unfortunately, contained an error.

In the original version of this article the first name of the coauthor was incorrectly spelled as "Khaled A. Alnaqbi". The correct spelling should have been "Khalid A. Alnaqbi". This is now presented correctly in this article.

The original article has been corrected.

The online version of the original article can be found at https://doi.org/ $10.1007 / \mathrm{s} 10067-018-4245-5$

Thurayya Arayssi

tha2002@qatar-med.cornell.edu

Elie A. Ak1

ea32@aub.edu.lb

Extended author information available on the last page of the article 


\section{Affiliations}

Thurayya Arayssi ${ }^{1}$ - Manale Harfouche ${ }^{2} \cdot$ Andrea Darzi $^{3} \cdot$ Samar Al Emadi $^{4} \cdot$ Khalid A. Alnaqbi $^{5} \cdot$ Humeira Badsha $^{6}$. Farida Al Balushi ${ }^{7}$. Carole $\mathrm{Dib}^{8}$ • Bassel Elzorkany ${ }^{9} \cdot$ Hussein Halabi ${ }^{10} \cdot$ Mohammed Hammoudeh $^{4}$. Wissam Hazer ${ }^{11}$. Basel Masri ${ }^{12}$. Mira Merashli ${ }^{13} \cdot$ Mohammed Omair $^{14} \cdot$ Nelly Salloum $^{8} \cdot$ Imad Uthman $^{13} \cdot$ Sumeja Zahirovic $^{1}$. Nelly Ziade ${ }^{15}$. Raveendhara R. Bannuru ${ }^{16}$. Timothy McAlindon ${ }^{16}$. Mohamed A. Nomier ${ }^{17}$ • Jasvinder A. Singh ${ }^{18,19}$. Robin Christensen ${ }^{20,21}$ • Peter Tugwell ${ }^{22}$ - Holger Schünemann ${ }^{23}$ • Elie A. Akl ${ }^{13,23,24}$

1 Department of Internal Medicine, Weill Cornell Medicine-Qatar, Doha, Qatar

2 Infectious Disease Epidemiology Group, Weill Cornell MedicineQatar, Cornell University, Qatar Foundation - Education City, Doha, Qatar

3 Faculty of Health Sciences, AUB GRADE Center, Clinical Research Institute, American University of Beirut, Beirut, Lebanon

4 Department of Internal Medicine, Hamad Medical Corporation, Doha, Qatar

5 Department of Rheumatology, Medical Institute, Al Ain Hospital, Al Ain, United Arab Emirates

6 Dr. Humeira Badsha Medical Center, Emirates Hospital, Dubai, United Arab Emirates

7 Rheumatology Unit, Royal Hospital, Muscat, Oman

8 Faculty of Health Sciences, American University of Beirut, Beirut, Lebanon

9 Faculty of Medicine, Cairo University, Cairo, Egypt

10 Rheumatology Division, Department of Internal Medicine, King Faisal Specialist Hospital and Research Center, Jeddah, Saudi Arabia

11 Nursing Department, Aspetar Qatar Orthopedic and Sports Medicine Hospital, Doha, Qatar

12 Department of Internal Medicine, Jordan Hospital, Amman, Jordan
13 Department of Rheumatology, American University of Beirut Medical Center, Beirut, Lebanon

14 Division of Rheumatology, College of Medicine, King Saud University, Riyadh, Saudi Arabia

15 Faculty of Medicine, Univeristé Saint Joseph, Beirut, Lebanon

16 Division of Rheumatology, Allergy and Immunology, Tufts Medical Center, Boston, MA, USA

17 Department of Epidemiology and Population Health, Faculty of Health Sciences, American University of Beirut, Beirut, Lebanon

18 Medicine Service, VA Medical Center, Birmingham, AL, USA

19 Department of Medicine at School of Medicine, and Division of Epidemiology at School of Public Health, University of Alabama at Birmingham, Birmingham, AL, USA

20 Musculoskeletal Statistics Unit, The Parker Institute, Bispebjerg and Frederiksberg Hospital, Copenhagen, Denmark

21 Department of Rheumatology, Odense University Hospital, Odense, Denmark

22 Department of Medicine, University of Ottawa, Ottawa, Canada

23 Department of Health Research Methods, Evidence, and Impact (HE\&I), McMaster University, Hamilton, ON, Canada

24 AUB GRADE Center, Clinical Research Institute, American University of Beirut, Beirut, Lebanon 